\title{
Docencia y Nuevas Formas de Aprendizaje en Universidades a Distancia en Iberoamérica
}

\author{
(The Training of Latin-American University Professors and New Ways \\ of Learning)
}

\author{
Miguel Casas Armengol \\ Universidad Nacional Abierta \\ (Venezuela)
}

\begin{abstract}
RESUMEN: Frente a los profundos e incesantes cambios del mundo actual, la universidad tradicional de cualquier país tiene que entrar pronta e irremediablemente a una reformulación integral de sus instituciones, sistemas y formas de aprendizaje. El proceso instruccional puede y debe diferir cada vez más, del paradigma educativo, que con muy pocos cambios hemos utilizado en la universidad durante muchos años y siglos. El aprendizaje, mediante métodos tradicionales, no resulta suficiente para desarrollar en los alumnos las capacidades cognitivas, creativas y organizativas, requeridas por la sociedad moderna; en efecto, el aprendizaje de hoy debe ir mucho más allá de la capacidad de recordar hechos, principios o procedimientos correctos, basados principalmente en información y memorización. Quienes aprenden deben, involucrarse especialmente en las áreas relativas a la creatividad, solución de problemas, análisis, evaluación, y satisfacer las necesidades de comunicación interpersonal así como la oportunidad de cuestionar, aportar y discutir. Ahora, el aprendizaje resulta cada vez más importante que la enseñanza y por otra parte, el vertiginoso y progresivo desarrollo de las Nuevas Tecnologías de Informática y Telemática, permiten fundamentales innovaciones en los procesos instruccionales, con especial relevancia para las instituciones con la Modalidad a Distancia. Lo anterior plantea la necesidad impostergable de reestructurar profunda y funcionalmente la formación de las futuras generaciones de Docentes de Universidades a Distancia.
\end{abstract}

Reestructuración Universitaria - Universidades a Distancia - Nuevas Formas de Aprendizaje Capacitación de Docentes Universitarios Iberoamericanos

\begin{abstract}
Facing the profound, accelerated and continuous changes of present world, the traditional universities of any society should enter promptly to an integral reestructuration of their institutions, systems and ways of learning. The instructional process could and should differ every time more from the traditional instructional paradigm that had been used in the universities for years and centuries. Learning through these traditional methods it is not sufficient to develop in the students the cognitive, creative and organisational capacities required by the modern society. Today learning becomes more important than teaching and furthermore, the whirling and continuous development off the information and telematic technologies, influence fundamental innovations in the instructional process, especially for Distance Education. All this considerations are basic for radical changes in the training of the future generation of professors for Distance Universities.
\end{abstract}

University Reconstruction - Distance Universities - New Ways of Learning - Training of LatinAmerican University Professors 
Durante más de doscientos años, los paradigmas de enseñanza usados en las universidades no experimentaron mayores cambios y aún hoy día, siguen predominando en la mayoría de las universidades existentes. Por otra parte, en el proceso educativo contemporáneo y quizás debido al fuerte peso de la rutina y la tradición, numerosos docentes le siguen atribuyendo mucha mayor importancia a la enseñanza que al aprendizaje.

La lenta evolución de estos procesos educativos no parecen tomar muy en cuenta los continuos y vertiginosos cambios de las estructuras sociales y científicas del mundo actual, que obligan a las universidades a emprender, sin mayores demoras, una profunda reconstrucción de sus estructuras, procesos y actores del dominio educativo.

El objetivo de este documento es examinar las razones de tales cambios y lo que ellos deben significar para la formación y ejercicio de los futuros docentes. En este análisis resulta indispensable estudiar con especial énfasis, las enormes posibilidades de utilizar funcionalmente, como herramientas de apoyo para la Educación a Distancia, las Nuevas Tecnologías, especialmente las que provienen de los campos de la Informática y la Telemática.

\section{FUERZAS QUE DETERMINAN LA NECESIDAD DE GRANDES CAMBIOS EDUCATIVOS}

El elemento mundial más importante que se observa en estas últimas décadas, ha sido el de un cambio incesante, acelerado, a veces inesperado y con frecuencia traumático. Él penetra casi todos los sectores sociales, políticos, científicos y tecnológicos, haciendo irrelevantes y obsoletos conceptos que por mucho tiempo se consideraron como indiscutibles. En esta situación, la universidad tradicional, que durante años y siglos ha tenido evoluciones muy lentas, se ve ahora forzada a emprender rápidamente una reestructuración integral de sus instituciones y sistemas, para así poder dar pronta respuesta a estas nuevas, múltiples y exigentes demandas. *1

Entre los factores que representan la orientación general de este proceso mundial de cambios y la necesidad de firmes respuestas educativas, señalaremos los siguientes:

Sociedades cada vez más informatizadas.

Dlobalización de la economía, mercados, información y educación.

\footnotetext{
* Como apoyo a las ideas y conceptos contenidos en este documento, se incluyen al final de algunos de sus párrafos, indicaciones numéricas (entre paréntesis), que permiten vincular tales ideas y conceptos específicos, con obras relevantes que los definen y amplían. Véase Referencias Bibliográficas.
} 
Amplio acceso y participación en la universidad, como antítesis del tradicional elitismo.

Duevas Tecnologías, especialmente Informática y Telemática.

Decnologías adecuadas a la Formación para el Trabajo y la Producción.

Duevas aproximaciones al Aprendizaje y a la Enseñanza.

Formación del Personal Académico para cumplir nuevos roles universitarios.

Variación de las formas y responsabilidades del Financiamiento universitario.

Casas (1995), señala otros factores adicionales, característicos en nuestro contexto socio político Iberoamericano:

Tendencias hacia la improvisación, contrarias a la previsión o planificación.

Grandiosos programas pero sin seguimiento ni evaluación.

Dolíticas sin continuidad al cambiar las autoridades.

Dingularidades del estudiante Iberoamericano.

Dscasa consolidación de una Cultura Tecnológica.

Dredominio del Titulismo versus el Conocimiento.

Resistencia a la Innovación.

Fuerte interferencia de factores del clientelismo político y de un sindicalismo exacerbado.

A lo anterior, se deben sumar otras limitaciones Internas Institucionales, presentes en muchas universidades Iberoamericanas:

Indefinición de la Direccionalidad Institucional, por ausencia de verdaderas Políticas.

Dstructuras Organizacionales inapropiadas para una gestión moderna.

D Insuficiente desarrollo de la Administración y la Gerencia eficientes.

Recursos Humanos limitados y diluidos entre muchas instituciones.

Todos estos factores deben encontrar respuestas categóricas e integradas en las nuevas estructuras y programas universitarios, pero en este documento, nos concentraremos en lo relativo a cómo ellos deben influir en la formación de los docentes, especialmente para la Educación a Distancia. 


\section{NUEVAS FORMAS DE APRENDIZAJE Y ENSEÑANZA}

Numerosos estudios e investigaciones, señalan ahora claramente que el proceso instruccional, puede y debe diferir cada vez más de las tradicionales prácticas educativas, que con muy pocos cambios hemos utilizado en la universidad durante muchos años ${ }^{* 2}$. El aprendizaje que usa exclusivamente métodos tradicionales, no resulta suficiente para desarrollar en los alumnos las capacidades cognitivas, creativas y organizativas, requeridas por la sociedad moderna y especialmente por sus nuevas demandas, señaladas en la sección anterior. En efecto, el aprendizaje de hoy debe ir mucho más allá de la capacidad de recordar hechos, principios o procedimientos correctos, basados principalmente en información y memorización. Si bien algunos de estos métodos siguen siendo necesarios, resultan mucho más importantes otros relativos a las áreas de creatividad, solución de problemas, análisis y evaluación. Quienes aprenden quieren satisfacer las necesidades de comunicación interpersonal y también la oportunidad de cuestionar, aportar y discutir. Se debe considerar el aprendizaje como una búsqueda individual de significado y relevancia, inserta en una actividad social e individual. Afirma Miller (1996, p. 38):

"La idea de la trascendencia del conocimiento por si mismo o por su propia importancia, está perdiendo parte de su atractivo dentro del proceso. En vez de esto, empezamos a comprender que el poder real se apoya en nuestra habilidad para buscar, analizar y usar críticamente la información, para hacer decisiones, resolver problemas y responder efectivamente a nuevas situaciones"

Conviene señalar algunos de los elementos y consideraciones que representan factores importantes, en lo relativo a la necesidad de nuevas formas para el aprendizaje y la enseñanza. Ellos son los siguientes:

En primer lugar, suponer que un futuro profesional pueda aprender al principio de sus estudios y en el nivel de Licenciatura (pre-grado), todo lo que él va a necesitar en el resto de su vida activa, es ignorar totalmente el mundo actual con su extraordinaria movilidad y complejidad, las cuales afectan casi todos los campos del saber. En muchas disciplinas el conocimiento pertinente se modifica substancialmente en menos de cinco años y esta tendencia continuará progresivamente. Algunas sociedades tienen planteada la necesidad de que la Licencia para ejercer profesionalmente, sea concedida sólo por algunos años y que ella deberá ser renovada mediante exámenes sucesivos que señalen inequívocamente la actualización y adquisición de los necesarios y nuevos conocimientos. Por consi-

\footnotetext{
* Esta observación tiene especial importancia para la Educación a Distancia, porque en ella el acelerado cambio experimentado durante las dos últimas décadas, en aspectos teóricos, metodológicos, tecnológicos, organizacionales, etc. no siempre resultan incorporados con la rapidez necesaria en algunas Universidades a Distancia Iberoamericanas, aparentemente atrapadas en una tradición de rutina e inmovilismo preocupantes.
} 
guiente, los Planes de Estudio deberán ser rediseñados y contemplar procesos continuos y de por vida para todas las carreras universitarias. Así mismo, las evaluaciones de los que estudian deberán hacerse más frecuentes para calificar competencias efectivas, más para el debido ejercicio, que para obtener certificados y diplomas, dentro de las nociones formales de escolaridad. ${ }^{3} 4$

Segundo, resulta cada vez más evidente que en el proceso instruccional, es más importante aprender que enseñar. Esta afirmación no pretende desestimar la validez de la enseñanza y de sus métodos, pero considerándola ahora como un apoyo que gira alrededor del aprendizaje. Por consiguiente, las Teorías de Aprendizaje, Aprender a Aprender (sustituyendo a los Métodos de Estudio), Solución de Problemas, Comunicaciones, Creatividad, Tecnologías Informativas, Autoevaluación, etc. adquieren una señalada y creciente importancia. ${ }^{5}$

Tercero, se ha constatado experimentalmente que no existe una sola "Inteligencia" sino "Múltiples Inteligencias" (musical, kinésica-corporal, matemáticalógica, lingüística, espacial, interpersonal, e intrapersonal) y ello tiene consecuencias en las diversas formas de aprender y de allí una razón más para darle importancia a las teorías sobre individualización del aprendizaje. ${ }^{6}$

Cuarto, el aprendizaje activo intenta lograr un "aprendizaje profundo" en vez del "aprendizaje superficial" característico de la enseñanza convencional, que estaba basada exageradamente en la memorización de la información, enfoque todavía predominante en muchos estudios universitarios profesionales. ${ }^{7}$

Quinto, la introducción de los procesos de interactividad, en sus diversas formas y posibilidades, facilita un aprendizaje dinámico y relevante que también permite una mayor individualización. ${ }^{8}$

Las características anteriores resultan igualmente importantes, tanto para la Modalidad Presencial como para la Modalidad a Distancia; lo mismo podríamos decir en lo relativo a las grandes Metas y Objetivos Finales de las instituciones universitarias que usan cualquiera de estas dos formas de enseñanza. La diferencia principal entre ambas modalidades se presenta en los Principios, Teorías, Procedimientos de Producción, Formas de Comunicación y Uso de ciertas Tecnologías. Por consiguiente, el docente efectivo en Educación a Distancia, debe poder dar respuesta también a los puntos anteriores, pero utilizando creativamente diferentes teorías, instrumentos y situaciones de aprendizaje.

\section{EL PERFIL DEL FUTURO DOCENTE EN EDUCACIÓN A DISTANCIA}

Antes de la profesionalización de la docencia universitaria en muchas universidades, especialmente de América Latina, el profesor universitario típico solía ser un destacado y experimentado profesional (ingeniero, médico ó abogado), 
que brindaba principalmente por razones de prestigio, algunas horas de su tiempo activo para formar a las nuevas generaciones. Para enseñar, él se valía de los conocimientos teóricos que había obtenido durante sus anteriores estudios profesionales de pre-grado, y de la experiencia acumulada a través de un ejercicio profesional intenso y extenso, que ocupó la mayoría de su tiempo.

$\mathrm{Su}$ actividad docente se expresaba principalmente mediante clases teóricas magistrales y unas pocas actividades prácticas o pasantías, según la naturaleza del curso y las limitaciones del tiempo y equipo disponible. La bibliografía de apoyo estaba constituida principalmente por los "apuntes" tomados en clase. Por consiguiente, el profesor representaba el "punto central para difundir la información y el conocimiento" para la especialidad correspondiente, agregándose también, la posibilidad de que él pudiera ejercer alguna influencia en sus alumnos, como "modelo" de esa profesión. La buena captación y memorización de las informaciones suministradas por este profesor, representaban las mejores credenciales para los alumnos considerados como sobresalientes, (el alumno destacado era el que poseía mayor capacidad de "absorción"). Para este "profesionalprofesor", las teorías y principios de la enseñanza, del aprendizaje y de la evaluación, resultaban irrelevantes y generalmente le eran poco conocidas.

Aunque las características anteriores están aún presentes en buena parte de nuestras universidades actuales (por su modelo más profesionalizante que educativo), sin embargo, ahora el "reclutamiento" del nuevo profesorado es muy diferente al del pasado. En efecto, al profesionalizarse la enseñanza universitaria muchos docentes jóvenes carecen de experiencia en la profesión respectiva, pero supuestamente compensan esta limitación, mediante postgrados, mejores laboratorios, actividades de investigación y mayor disponibilidad de libros, revistas y equipos. Sin embargo, en lo referente a su aporte directo al proceso de aprendizaje, el "paradigma educativo tradicional" que les sirve de apoyo principal, sigue siendo generalmente el de la Clase Magistral ("pizarrón y tiza"), la Información y la Memorización. Aunque algunos educadores y psicólogos innovadores han tratado de romper esta "esclavitud disfuncional", sólo explicable por el peso de la tradición y la rutina docentes, ni las investigaciones recientes ni el gran potencial de las nuevas tecnologías, han logrado hasta ahora una masiva y significativa aceptación y utilización, así como tampoco, se ha podido impulsar, la implantación de profundas reestructuraciones institucionales, para una universidad como la actual, en una situación de extraordinarios y constantes avances mundiales. El Profesor universitario que no ha podido superar el perfil tradicional descrito en los párrafos anteriores, ha perdido total vigencia en la universidad actual y futura. Ello no significa que la función docente y los docentes sean ahora menos importantes; por el contrario, estas funciones adquieren sin duda una proyección distinta, pero mucho más trascendente y compleja. Porque aunque él profesor ya no seguirá siendo el único "faro" de información, las nuevas teorías, enfoques y herramientas tecnológicas sí le permitirán dar aportes más efectivos al proceso de 
aprendizaje así como a su individualización. En otras palabras, el nuevo docente deberá orientarse hacia la guía, estímulo y evaluación de estudiantes, que ya no se conformarán con un saber restringido, encapsulado, estático y mecánico. Las nuevas generaciones estudiantiles no pueden memorizar e incorporar pasivamente limitados puntos de vista que ellos no pueden discutir y apreciar desde diferentes ópticas. El cúmulo de ideas, informaciones y hechos que suceden a escala mundial, así como las variadas y cambiantes tecnologías actuales requieren, que el profesor asuma hoy día un rol muy distinto del que pretendía presentar unas pocas verdades dogmáticas..$^{9,10}$

En la realidad actual y con las avanzadas tecnologías disponibles, cualquier alumno puede acceder fácilmente a informaciones y hechos, mucho más amplios, profundos, relevantes y actualizados, que los que solía comunicar el profesor tradicional. Por consiguiente, el nuevo rol del profesor debe procurar la orientación del alumno para que él encuentre caminos que lo beneficien en su búsqueda del conocimiento. Por otra parte, el nuevo docente debe plantear formas originales para que sus alumnos desarrollen la observación, la creatividad y la solución de problemas, referidos a la especialidad estudiada, pero siempre insertos en un amplio y real contexto social, científico y tecnológico. ${ }^{11}$

Como ejemplo de lo que puede representar el "Aprendizaje Efectivo", aplicado a la formación de Ingenieros, transcribimos (Letelier, 1997, p. 95) el conjunto de comprensión de materias y logro de habilidades de los alumnos respectivos:

I “Comprensión de los principios científicos o conceptuales que gobiernan determinados temas.

II Capacidad de aplicar el conocimiento en problemas relacionados con la realidad en que se desenvuelve la profesión respectiva.

III Capacidad de asociar las materias estudiadas con otras disciplinas y áreas de problemas.

IV Capacidad de extender el conocimiento adquirido a través de la respuesta a preguntas bien formuladas por el alumno mismo y a través de otros recursos creativos.

La experiencia enseña que un aprendizaje efectivo se logra cuando la docencia respeta, por lo menos, los siguientes requisitos:

V Entrega de conocimientos de acuerdo a las estructuras conceptuales con que inician los alumnos su estudio. Debe haber un empalme eficiente entre lo que los alumnos saben y aquello que se les va a entregar.

VI La enseñanza propende a un aprendizaje asociativo. Este se caracteriza por relacionar los conceptos más importantes con otros conceptos que faciliten su aplicación y su interrelación con otras áreas. 
VII El avance del proceso de aprendizaje tiene el ritmo adecuado para que las asociaciones y aplicaciones tengan lugar.

VIII En general se enseñan pocas materias con profundidad y a un ritmo que da lugar al dominio de principios y conceptos básicos, a las asociaciones y a las aplicaciones."

Para lograr los objetivos anteriores el nuevo Docente en Educación a Distancia debe tener un perfil que se apoye principalmente en cuatro sólidas bases:

D Conocimiento profundo, actualizado y teórico-práctico de su disciplina, (física, química, sociología, ingeniería, etc.), incluyendo investigaciones más relevantes.

Dominio de Principios, Teorías y Metodologías de la Educación a Distancia.

- Manejo efectivo de las Nuevas Tecnologías Informativas y Telemáticas y de su utilización funcional, tanto en el proceso de aprendizaje como en la aplicación a su disciplina.

Claridad sobre los fines educativos universitarios y capacidad para la formulación de originales estrategias instruccionales. ${ }^{12}$

\section{LAS NUEVAS TECNOLOGÍAS EN LA FORMACIÓN DE DOCENTES}

Entre las variadas influencias que justifican y permiten transformar el rol del nuevo docente universitario, se destacan ahora por su extraordinaria y creciente importancia, un conjunto de Modernas Tecnologías, principalmente las relativas a Informática y Telemática. Pero conviene aclarar inicialmente lo que entendemos por tecnología a fin de disipar la popular y errónea creencia de que ésta se refiere exclusivamente a la adquisición y uso de equipos, redes y software. Para ello usaremos la definición siguiente sobre Tecnología (Naughton, 1994, p. 12):

"Aplicación del conocimiento científico y de otros conocimientos, a problemas concretos, mediante un conjunto integrado de estrategias, procesos y tareas prácticas, llevadas a cabo por organizaciones que incluyen personas y equipos (tanto tradicionales como modernos)"

Aprender, aplicar y enseñar las tecnologías actuales en el Proceso Educativo es una tarea importante pero compleja, que debe formar parte indispensable de la preparación y de las herramientas del nuevo docente y especialmente del docente en Educación a Distancia, dada la extraordinaria utilidad de tales tecnologías para resolver muchos de los problemas característicos de esta Modalidad Educativa. Sin embargo, no resulta sencillo ni inmediato, convencer a la generación actual de docentes de la necesidad de aprestarse a conocer y manejar con 
efectividad esta extraordinaria herramienta, cada vez más poderosa y versátil. Casas (1997, p. 60) expresa al respecto:

"la mayor barrera para la aceptación de las innovaciones educativas, y el uso de nuevas tecnologías, dentro de las universidades, no es la falta de recursos, o la poca voluntad de sus directivos para aceptar las necesidades de cambio, u objeciones ideológicas o filosóficas; en realidad, el mayor obstáculo es el temor de muchos profesores que no se sienten cómodos con las innovaciones tecnológicas o quizás lo más importante, que no saben como usarlas efectivamente (Bates, 1995). El problema no se reduce a carencia de entrenamiento para una tecnología específica, sino la falta de una estructura conceptual apropiada para guiar el uso de la tecnología. En otras palabras, tanto en el contexto cultural como en el institucional, no está debidamente consolidada una "Cultura Tecnológica". Por consiguiente, esto significa que muchas personas con responsabilidades de enseñanza, no han recibido una formación instruccional apropiada para basar su práctica docente e investigativa."

El tema de las Nuevas Tecnologías para la Educación recibió una considerable atención en la última Conferencia del International Council on Distance Education (ICDE), celebrado en PennState University U.S. (1997). Hubo abrumador acuerdo en que para la Educación a Distancia el uso funcional de tales tecnologías resulta ahora de especial e inevitable importancia. Sin embargo, se advirtió que el empleo en forma aislada de estos modernos equipos (computadores, redes, vídeo, audio, etc.) sólo como suplemento de formas tradicionales de instrucción, desperdicia su gran potencial y no hace más que agregar costos adicionales a la función tradicional de enseñanza - la clase magistral - que ya resulta de por sí onerosa. Por el contrario, la mejor utilización de estas tecnologías, se logra mediante enfoques integrados de programas de computación, sistemas y redes que permitan el cabal aprovechamiento de la informática y de la telemática. ${ }^{13,14,15}$.

Mingle (1995), considera que muy probablemente tenderemos hacia el desarrollo de infraestructuras nacionales de información que pronto se convertirán en infraestructuras internacionales, y donde todo ello, requerirá un repensar de muchas de las suposiciones que las universidades han utilizado como punto de partida para su gestación. Todas estas consideraciones refuerzan la tesis de que ya estamos llegando a lo que se denomina "el fin de las universidades autocontenidas y aisladas" y el inicio y vigoroso desarrollo de Federaciones, Alianzas Estratégicas y Sistemas Universitarios, extendidos nacional e internacionalmente. ${ }^{16,17} \mathrm{El}$ mismo Mingle, percibe como influencias futuras facilitadas por el desarrollo de las nuevas tecnologías, puntos como:

Da distinción actual entre "instrucción en el Campus" y la que se obtiene en la "educación a distancia", tenderá a desaparecer, porque los estudiantes usarán los mismos elementos — por ejemplo la conferencia con computadores -, no importa donde estén. En este sentido conviene reconocer la notable fuerza del movimiento mundial denominado "Convergencia", que consiste en la incorporación progresiva de la modalidad a distancia en sectores importantes de grandes universidades presenciales. 
D Tanto el curriculum como las formas de entrega de la instrucción, pondrán el contenido académico en el contexto del "mundo real", especialmente en la casa y en el lugar de trabajo. Aprendizaje, trabajo, familia y vida social, concurrirán en una era de "aprendizaje durante toda la vida".

D La Tecnología creará un amplio mercado para Educación Superior que le dará a los estudiantes mayores posibilidades de escogencia e individualización. Ellos podrán decidir, asesorados por sus tutores, donde estudiar y como mezclar e integrar cursos, escogiendo entre distintas instituciones proveedoras, tanto del mismo país como de otros países.

D La efectividad de los sistemas de entrega educativa será medida principalmente por criterios centrados en el que "aprende".

Ampliando la idea del "uso integrado de medios", deben citarse algunos planteamientos hechos en Francia, Canadá y más recientemente en Inglaterra. Los Canadienses han esbozado la aparición de una nueva especialidad, que denominan "Mediática" o "Telemediática". Por su parte Eisendstadt (1995) en Inglaterra, ha acuñado para ese enfoque, el nombre de "Medios para el Conocimiento" ("Knowledge Media"). En todos los casos, estos enfoques y nombres, emergen como resultado de la convergencia entre computación, telecomunicaciones y las ciencias cognoscitivas. Para algunos autores esta convergencia es también considerada, como la "Tercera Generación" de las tecnologías con grandes posibilidades inmediatas de aplicación a la Educación a Distancia. ${ }^{18}$

Por otra parte, (según Casas, 1987) la evolución de la Tecnología Educativa en los últimos años ha pasado por tres etapas bien diferenciadas, de las cuales la última resulta especialmente relevante para la Educación a Distancia. La Primera y más rudimentaria — conocida generalmente como "Audiovisualismo" —, se refería al uso esporádico de algunas ayudas audio/visuales y aparatos, que permitían, generalmente por iniciativas individuales del profesor, enriquecer las exposiciones orales de sus clases magistrales. En una Segunda Etapa, el concepto de sistemas, introdujo la posibilidad de que especialistas en tecnología educativa colaboraran con los profesores de diversas materias para organizar y sistematizar los procesos totales de enseñanza-aprendizaje y los ayudaran a clarificar sus objetivos, mejorar sus métodos y evaluar las experiencias y resultados; en otras palabras, su objetivo principal era apoyar la actividad del profesor y de sus enseñanzas. Pero es en la tercera Etapa de esta evolución, donde ahora se plantea cómo la tecnología educativa debe apoyar más directamente al estudiante en su proceso de aprendizaje, cuestión ésta de fundamental importancia para quienes siguen cursos de educación a distancia.

Chacón (1997) resume las características distintivas de los sistemas educativos que emergen de la revolución de las nuevas tecnologías de información y comunicación: 
Interactividad. Referida tanto a la interacción "persona-computador" como "persona-persona"; está presente en los programas tutoriales en CD-ROM, simulaciones, aprendizaje colaborativo en red, correo electrónico, teleconferencias, etc.

Aprendizaje centrado en procesos. Que reconoce la imposibilidad actual de trasmitir en un curso - aún de Postgrado-, el cúmulo de informaciones existentes. Por ello, la actividad de los cursos debe centrarse más en los procesos para generar y utilizar la información, que en el cuerpo de información en sí mismo. Estos procesos son, por ejemplo: observación, análisis, integración, comparación, evaluación, etc.

Globalización. La información de cualquier parte del mundo está disponible al toque del teclado. La Universidad debe abrir sus fronteras a todas las fuentes disponibles. Esto también impacta las tradicionales nociones de autoridad académica, basada en el profesor o el experto. Ahora cualquiera puede sorprender al profesor o al experto con nuevas informaciones. El liderazgo debe estar basado en la capacidad del docente para orientar, promover actividades reflexivas y ayudar a los que tienen dificultades.

Redes de Conocimiento. El uso extendido de Internet para la diseminación de informaciones es la creación de redes vivientes de conocimiento, en las cuales cada aspecto de una disciplina es estudiado por distintos equipos de investigadores, que preparan resúmenes o artículos y los hacen disponibles a través de la red. ${ }^{19}$

Ambientes Sintéticos Compartidos. El desarrollo combinado de técnicas de Simulación, Inteligencia Artificial y Robótica ha permitido el diseño de modelos virtuales de aprendizaje o robots manipulables por computador, en los cuales muchas operaciones que son costosas, riesgosas o difíciles de llevar a cabo en el mundo real, pueden ser cumplidas ahora en la tranquilidad de un mundo virtual. De esta manera el estudiante puede "aprender haciendo" muchas cosas que antes sólo podía "aprender oyendo o leyendo".

Para resumir y clasificar en forma muy general la enorme variedad de modernos medios y equipos disponibles, especialmente para la educación a distancia, los agruparemos en dos categorías: A) Medios para la enseñanza tipo "Clase Remota", generalmente "sincrónicos"; y B) Medios para la enseñanza "Flexible y Personal" generalmente "asincrónicos").

Entre los primeros (A) citaremos: Audio-Teleconferencia; Audio Teleconferencia con una posible segunda red de comunicaciones, usada para transmitir Gráficos (conocida también como Audiográficos); Teleconferencia usando computadores(que puede incluir imágenes, color, movimiento y sonido; Internet, usando especialmente programas en línea; Televisión "Slow Scan"; Vídeo Conferencia, 
con líneas separadas de Audio para preguntas; Vídeo Conferencia, entre múltiples puntos, en cada uno de los cuales existe recepción y emisión (la tecnología más útil para asesorías, seminarios y conferencias, pero la de mayor costo); etc.

Entre los segundos (B) destacaremos el "aprendizaje flexible" o "aprendizaje con múltiples canales". El objetivo principal es aumentar o reemplazar las actividades de un salón convencional, mediante un amplio grupo de tecnologías, tales como Multimedios, Multimedios interactivos, Correo Electrónico, cursos desarrollados mediante computación, etc. Las fuerzas principales de esta aproximación son la flexibilidad y la interactividad. En muchos casos una determinada estrategia Instruccional puede combinar los Medios (A) con los Medios (B).

\section{SÍNTESIS DE CONCLUSIONES}

D En un mundo como el actual, caracterizado por profundos y vertiginosos cambios en todos los órdenes social, político, científico, tecnológico, profesional, etc., una institución universitaria conservadora que pretenda aferrarse a la lenta evolución de otras épocas, enfrentará una disfuncionalidad cada vez más inaceptable y hasta destructiva. Las fuerzas mundiales, nacionales y regionales que determinan la inevitabilidad de grandes cambios universitarios requieren procesos integrales de reestructuración, imposibles de lograr con simples, y lentos perfeccionamientos aislados.

D El aprendizaje usando exclusivamente métodos tradicionales — clase magistral, informaciones, memorización, apuntes, etc.- no resulta suficiente para desarrollar en los alumnos las capacidades cognitivas, creativas y organizativas, requeridas por la sociedad contemporánea. En el proceso instruccional moderno resulta más importante aprender que enseñar. Diversos y fundamentales factores ameritan sustanciales e inmediatos cambios en las estructuras universitarias y especialmente en sus formas de aprendizaje y enseñanza.

D La emergencia y revolucionario desarrollo de Nuevas Tecnologías de Información y Comunicaciones, imprimen características distintivas y ofrece extraordinarias posibilidades para nuevas formas de aprendizaje y enseñanza. Sin embargo, el uso funcional de estos procesos e instrumentos requiere la consolidación de una "Cultura Tecnológica" y una especial preparación que por ahora tienen muy pocos docentes universitarios de las actuales generaciones.

D El reclutamiento y formación de los futuros docentes para universidades presenciales y a distancia, debería fundarse en la adecuada respuesta a los principales problemas planteados en este documento. La diferencia prin- 
cipal entre los docentes para estas dos modalidades - presencial y a distancia-, no reside en las grandes metas y objetivos de sus universidades, sino en las teorías, principios, metodologías y tecnologías aplicables a sus respectivos procesos instruccionales. Sin embargo, se prevé que en un proceso de "Convergencia" las diferencias entre ellas, disminuirán progresivamente. Por ahora, en el caso de la Educación a Distancia, los problemas principales a resolver, son los relativos a la falta de contigüidad física entre docentes y discentes y a la individualización del aprendizaje.

\section{REFERENCIAS BIBLIOGRÁFICAS}

Bates, A.W. (1995). Technology, Open Learning and Distance Education. London: Routledge.

Casas Armengol, M. (1987). Universidad sin Clases. Educación a Distancia en América Latina. Caracas: Kapelusz/UNA. (1995). Distance Education Universities in Latin-America: expectations and disappointments. En: One World, Many Voices. Quality in Open and Distance Learning. Ed. David Sewart. Anales de la XVII Conference for Distance Education. ICDE. Vol 1. Birmingham, 1995.

(1997). "Reingeniería de la Educación Superior Venezolana". Asuntos. Año 1, № 1. Marzo. CIED. Centro Internacional de Educación y Desarrollo. Caracas.

Сhacón, F. (30 de Mayo de 1997). Mensaje Electrónico enviado en relación con la Conferencia "Redonda" y sobre el tema de "Características distintivas de los Sistemas Educativos que emergen de la revolución de las nuevas tecnologías de información y comunicación". Caracas.

EIsenstadT, M. (1995). "Over Strategy for Global Learning”. Times Higher Educational Supplement, Multimedia Section, 7 April, vi-vii. London.

Letelier, Mario (1997). "Calidad y pertinencia de la educación en ingeniería: Un enfoque evolutivo”. En: Evaluación y Acreditación Universitaria. Metodologías y Experiencias. Eds. Eduardo Martínez/Mario Letelier. Caracas: Nueva Sociedad - UNESCO.

MiLLER, Gary (1996). Technology, the curriculum and the learner: Opportunities for open and distance education. En: Roger Mills \& Alan Tait Eds.: Supporting the Learner in Open and Distance Learning. London: Pitman Publishing.

Mingle, J. R. (1995). "Vision and Reality for Technology-based Delivery Systems in Postsecondary Education". Governors' Conference of Higher Education (St. Louis, Missouri). Denver: State Higher Education Executive Officers.

Naughton, John (1994). "What is technology?" En: (Frank Banks ed.) Teaching Technology. London: Routledge - Open University.

1 Revista Asuntos CIED. 1997. Año 1. N 1. Caracas.

2 Clark, Burton R. (1962). Educating the expert society. San Francisco: Chandler.

${ }^{3}$ Houle, Cyril O. (1989). Continuing Learning in the Professions. San Francisco: The JosseyBass. 
${ }^{4}$ Maclure, Stuart (Ed.) (1991). Learning to think: thinking to Learn. Oxford: Pergamon Press.

${ }^{5}$ Gardner, Howard (1993). Multiple Intelligences. The Theory in Practice. New York: Basic Books.

6 Gross, Ronald (1991). Peak Learning. New York: Putman.

${ }^{7}$ Ruiz I Bel (1988). Técnicas de Individualización Didáctica. Madrid: Cincel-Kapelusz.

8 Phillis, D.C. \& Soltis, Jonas F. (1991). Perspectives on Learning. New York: Teachers College.

${ }_{9}$ NovaK, Joseph D. \& Gowin, D. Bob (1993). Learning how to learn. Cambridge: Cambridge University Press.

10 WiLson, John D. (1981). Student Learning in Higher Education. London: Croom Helm.

11 Dill, David D. and Associates (1991). What Teachers Need to Know. San Francisco: Jossey-

Bass,

12 ICDE - Pennstate (1997). The New Learning Environment. A Global Perspective. CD.ROM conteniendo los documentos presentados a la $18^{\text {a }}$ Conferencia Mundial del ICDE. Junio 26. State College.

13 Lockwood, F. (Ed.) (1995). Open and Distance Learning Today. London: Routledge studies in Distance Education.

${ }^{14}$ Daniel, John S. (1996). Mega-Universities and Knowledge Media. Technology Strategies for Higher Education. London: Kogan Page.

15 Tiffin, John \& Rajasingham, Lalita (1995). In Search Of The Virtual Class. Education in an Information Society. New York: Routledge.

16 Model Virtual Universities (1997). En: Revista Education at a Distance. United States Distance Learning Association (USDLA). January. Volume 11\# 1. San Ramon.

17 Unesco/Cresalc (1992). Calidad, Tecnología y Globalización en la Educación Superior Latinoamericana. Caracas: Cresalc.

18 Forsyth, Ian (1996). Teaching and Learning Materials and the Internet. London: Kogan Page. 19 Portway, Patrick S. \& Lane, Carla (1997). Guide to Teleconferencing and Distance Learning.. USDLA. San Ramón: Applied Business teleCommunication.

\section{PERFIL ACADÉMICO DEL AUTOR}

Miguel Casas Armengol es Arquitecto (Universidad Nacional Colombia; Universidad Central Venezuela). Ph.D. en Educación (Stanford University: California, U.S.A.). Profesor Titular de la Universidad del Zulia, Maracaibo y de la Universidad Nacional Abierta, Caracas. Rector Fundador de la Universidad Nacional Abierta. Investigador Visitante del Instituto de Planificación Educativa, UNESCO, París, y de la Universidad de Stanford, California. Autor de: Universidad sin Clases y Coautor (Capítulos) en siete libros de Pergamon Press y de Unesco.

Líneas de Investigación: Universidad y Desarrollo Regional y Nacional. Organización y Modernización de Universidades a Distancia. Nuevas Tecnologías en Educación Superior a Distancia.

Prof. Miguel Casas Armengol (Ph.D)

Residencias Samana Ap 3-A. Av. Los Samanes.

La Florida. Caracas 1050.VENEZUELA

Telfs. (58-2) $781.40 .51 / 793.68 .02$

Correo Electrónico: mcasas@iusnet.net 